\title{
Palynomorphological peculiarities of representatives of tribes Lindenbergieae and Cymbarieae and pollen evolution in early-branching lineages of Orobanchaceae
}

\author{
Zoya M. TSYMBALYUK, Sergei L. MOSYAKIN \\ M.G. Kholodny Institute of Botany, National Academy of Sciences of Ukraine \\ 2 Tereshchenkivska Str., Kyiv 01004, Ukraine \\ palynology@ukr.net
}

Tsymbalyuk Z.M., Mosyakin S.L. Palynomorphological peculiarities of representatives of tribes Lindenbergieae and Cymbarieae and pollen evolution in early-branching lineages of Orobanchaceae. Ukr. Bot. J., 2018, 75(2): 123-136.

\begin{abstract}
Pollen morphology of four species belonging to four genera of the tribe Cymbarieae and two species of Lindenbergia of the tribe Lindenbergieae (Orobanchaceae) was studied using light and scanning electron microscopy. Pollen grains in Lindenbergieae are 3-colporate (rarely 2-colporate), prolate, spheroidal and oblate-spheroidal, small-sized, with reticulate exine sculpture. Pollen grains in Cymbarieae are 3-colpate, rarely 4-colpate, prolate, spheroidal and oblate-spheroidal, medium-sized. Exine sculpture in Cymbarieae is retipilate and rugulate-retipilate. Pollen characters of Cymbarieae and Lindenbergieae are compared with pollen patterns in Paulowniaceae and crown clades of Orobanchaceae. The earliest-branching clade of Orobanchaceae (Lindenbergieae) is palynomorphologically similar to Paulowniaceae (the clade sister to all Orobanchaceae) and to Wightia. These palynomorphological findings confirm the phylogenetic patterns recently revealed in basal Orobanchaceae and their closest relatives. It is concluded that the colporate (most probably 3-colporate) type of pollen grains could be ancestral in Orobanchaceae. Pollen diversity in crown clades of Orobanchaceae evolved on the base of a few pollen types and subtypes, which were peculiar to hypothetical ancestors of Orobanchaceae and are probably preserved in the extant taxa of Paulowniaceae and Lindenbergieae.
\end{abstract}

Keywords: Cymbarieae, Lindenbergieae, Orobanchaceae, pollen evolution, pollen morphology, phylogeny, taxonomy

\section{Introduction}

The tribe Cymbarieae D. Don (Orobanchaceae Vent.) in its current circumscription includes six or seven genera and ca. 14 species of root hemiparasites (Fischer, 2004; Bennett, Mathews, 2006; Reveal, 2012; McNeal et al., 2013; Olmstead, 2016). The genera currently included in the tribe are Bungea C.A. Mey. (2 species, southwestern and Central Asia and China), Cymbaria L. sensu lato (4 species, southeastern Europe, Central and eastern Asia; many authors also include here Cymbochasma (End1.) Klokov \& Zoz), Lesquereuxia Boiss. (1 species, L. syriaca Boiss. \& Reut. = Siphonostegia syriaca (Boiss. $\&$ Reut.) Boiss., Eastern Mediterranean; most probably nested in Siphonostegia: see McNeal et al., 2013), Monochasma Maxim. ex Franch. \& Sav. (4 species, East Asia), Schwalbea L. (1 species, S. americana L., eastern North America), and Siphonostegia Benth. (2 species: $S$. chinensis Benth. and S. laeta S. Moore, East Asia; or 3 species, if $S$. syriaca is included) (Fischer, 2004; Bennett, Mathews, 2006; Takhtajan, 2009; McNeal et al., 2013; Olmstead, 2016).

(c) Z.M. TSYMBALYUK, S.L. MOSYAKIN, 2018
The genus Lindenbergia Lehm. is represented by non-parasitic taxa and includes $12-15$ species occurring mostly in tropical regions, from northeastern Africa through southern and southeastern Asia (including the Himalayas) to Philippines (Mabberley, 1997; Stevens, 2001-onwards; Fischer, 2004; Olmstead, 2016).

In earlier variants of the system of A.L. Takhtajan (1987, 1997) the mentioned genera Schwalbea, Bungea, Siphonostegia, Cymbaria, and Monochasma were included (with many other genera) in the tribe Rhinantheae Lam. \& DC. of Scrophulariaceae subfam. Rhinanthoideae. In the latest version of his system, Takhtajan (2009) placed the genera Schwalbea, Cymbaria (including Cymbochasma), Siphonostegia, Lesquereuxia, Bungea, Monochasma in the tribe Cymbarieae (also with some other genera), and their familial and subfamilial placement remained the same. Fischer (2004) included in Cymbarieae six genera: Schwalbea, Cymbaria (including Cymbochasma), Siphonostegia, Lesquereuxia, Bungea, and Monochasma.

Takhtajan $(1987,1997)$ initially placed Lindenbergia in Scrophulariaceae subfam. Scrophularioideae trib. Gratioleae Benth. In 2009 he moved that genus to the 
tribe Stemodieae Reveal, which was positioned in his system close to Gratioleae, in the same subfamily. Fischer (2004) placed Lindenbergia in Scrophulariaceae trib. Stemodieae. Now the genus is placed in Orobanchaceae trib. Lindenbergieae T. Yamaz. (= Lindenbergiaceae Doweld, 2001).

Many authors studied and discussed pollen morphology of representatives of Scrophulariaceae and Orobanchaceae in general (Minkin, Eshbaugh, 1989), or specifically Rhinantheae (Inceoğlu, 1982; Lu et al., 2007; Tsymbalyuk, Mosyakin, 2017), and its taxonomic and evolutionary significance (see also Tsymbalyuk, Mosyakin, 2013a, b; Mosyakin, Tsymbalyuk, 2015a, b, 2017 and references therein). However, there are only a few publications reporting data on pollen of taxa now placed in Cymbarieae; these publications provide some data based on light and scanning electron microscopy (Inceoğlu, 1982; Minkin, Eshbaugh, 1989; Lu et al., 2007). Pollen grains of two species (Bungea trifida and Lesquereuxia syriaca) currently placed in Cymbarieae were also studied using transmission electron microscopy (Inceoğlu, 1982). However, no comprehensive analysis of palynomorphological peculiarities of Cymbarieae in its updated phylogeny-based circumscription has been done until now.

Prijanto (1969) provided the following information about pollen grains of Lindenbergia: "Pollen grains small, 3-colporate, rarely 2-colporate, oblate spheroidal to prolate spheroidal (polar axis 12-19 $\mu \mathrm{m}$ long, equatorial diameter 13-18 $\mu \mathrm{m}$; apocolpium diameter $3-5 \mu \mathrm{m}$; exine about $0.8-1.5 \mu \mathrm{m}$ thick, sexine as thick as nexine, reticulate". However, no images of pollen grains were published by Prijanto (1969). Hjertson (1995) updated the information of Prijanto (1969) and provided SEM images of pollen grains of $L$. muraria (Roxb. ex D. Don) Brühl and L. grandiflora (Buch.Ham. ex D. Don) Benth. He concluded that "pollen grains in Lindenbergia are tricolporate, oblate to prolate in shape, with a 12-19 $\mu \mathrm{m}$ long polar axis and an equatorial diameter of $13-18 \mu \mathrm{m}$. The exine as well as the sexine is about $1 \mu \mathrm{m}$ thick, and the surface is reticulate".

Molecular phylogenetic evidence indicate that Cymbarieae is one of the early-branching (basal) lineages of Orobanchaceae, most probably its secondbranching clade, following the basalmost clade of Lindenbergia (Orobanchaceae trib. Lindenbergieae = Lindenbergiaceae) (McNeal et al., 2013; Cusimano, Wicke, 2016; Schneider et al., 2016; Schneider, Moore, 2017 , etc.). According to molecular phylogenetic studies (Bennett, Mathews, 2006; McNeal et al., 2013), the clade of Cymbarieae is subdivided into two subclades: one of these clades includes Bungea, Cymbaria (including Cymbochasma), and Monochasma; the other houses Siphonostegia (including Lesquereuxia) and Schwalbea.

Our earlier studies and analysis of pollen morphology in basal clades of Scrophulariaceae sensu stricto allowed us to outline the main trends of morphological pollen evolution in the family in its new circumscription and to hypothesize on possible ancestral pollen types in the group (Mosyakin, Tsymbalyuk, 2015, 2017). Because of that, we may expect that a comprehensive analysis of pollen morphology in early-branching clades of Orobanchaceae could bring comparable results and shed light on main trends of pollen evolution in that group as well.

The purpose of the present research was to study and analyze the morphological features of pollen grains of representatives of Cymbarieae and Lindenbergieae in the updated taxonomic circumscriptions of these tribes, and to compare the pollen patterns with existing systems and molecular phylogenetic data.

\section{Materials and methods}

Pollen of four species belonging to four genera of Cymbarieae (Bungea, Cymbaria, Cymbochasma, and Siphonostegia) was sampled in the National Herbarium of Ukraine (KW - herbarium of the M.G. Kholodny Institute of Botany, National Academy of Sciences of Ukraine, Kyiv, Ukraine). Pollen grains of two species of Lindenbergia were sampled in the herbarium of the Missouri Botanical Garden (MO; St. Louis, Missouri, USA). Data of the studied specimens are cited exactly according to the label information, in English translation and in original languages.

In general, the methods used in the present study are essentially the same as those we used earlier (see Mosyakin, Tsymbalyuk, 2015a, b, 2017). Pollen morphology was studied using light microscopy and scanning electron microscopy. For light microscopy studies (LM, Biolar, $\times 700)$, the pollen was acetolyzed following Erdtman (1952). For size determinations, 20 measurements were taken along the polar $(\mathrm{P})$ and equatorial (E) axes for each species. For scanning electron microscopy (SEM, JSM-6060LA), pollen grains were treated with $96 \%$-ethanol, then these samples were sputter-coated with gold and investigated at the Center of Electron Microscopy of the 
M.G. Kholodny Institute of Botany. The micrographs were minimally edited with Adobe Photoshop 6.0 to enhance the images.

Terminology used in descriptions of pollen grains mainly follows the glossaries by Tokarev (2002) and Punt et al. (2007) with some necessary minor adjustments.

\section{General description of pollen grains}

\section{Lindenbergieae}

Pollen grains in monads, radially symmetrical, isopolar, 3-colporate (rarely 2-colporate, according to Prijanto, 1969), prolate, spheroidal or oblatespheroidal; mainly small-sized: $\mathrm{P}=13.3-18.6 \mu \mathrm{m}$ (12-19 $\mu \mathrm{m}$, according to Prijanto, 1969), $\mathrm{E}=13.3-$ $18.6 \mu \mathrm{m}$. Outline in polar view 3-lobate, in equatorial view elliptical or circular. Colpi long, narrow, 1.3$2.7 \mu \mathrm{m}$ wide, with mainly indistinct (sometimes distinct) more or less strict margins, with acute ends. Endoapertures mainly indistinct, circular, 2.4$2.7 \mu \mathrm{m}$ long and wide. Exine 0.7-1.6 $\mu \mathrm{m}$. Tectum nearly equal to infratectum. Columellae indistinct or distinct, thin, or exine layers invisible. Exine sculpture reticulate. Colpus membrane smooth.

\section{Cymbarieae}

Pollen grains in monads, radially symmetrical, isopolar, mainly 3-colpate, rarely 4-colpate, prolate, spheroidal, or oblate-spheroidal. The outlines in equatorial view elliptical or circular, in polar view sub-circular, circular, and sub-triangular; medium- or large-sized: $\mathrm{P}=22.6-47.9 \mu \mathrm{m}$ (up to $50 \mu \mathrm{m}$, according to $\mathrm{Lu}$ et al., 2007), $\mathrm{E}=25.3-43.9 \mu \mathrm{m}$. Colpi long or medium-length, narrow, medium-width to wide, 1.3$6.6 \mu \mathrm{m}$ wide, with indistinct, uneven margins, and blunt, acute, or indistinct ends. Exine 0.7-3.3 $\mu \mathrm{m}$. Tectum nearly equal to infratectum, columellae mainly indistinct or thin and arranged more or less regularly. Exine sculpture retipilate, rarely rugulate-retipilate. Capita (pila heads) arranged in circle in most or all regions of pollen surface. Caput of pila $0.16-0.67 \mu \mathrm{m}$ (up to $0.90 \mu \mathrm{m}$, according to $\mathrm{Lu}$ et al., 2007). Colpus membrane granulate.

\section{Pollen types and subtypes}

Based on their aperture types, pollen grains of the studied species belong to the same basic pollen types. The second type (3-colpate) contains four subtypes segregated according to their exine sculpture, pollen size, details of colpi, and thickness of the exine.
Type I - 3-colporate

Sculpture reticulate.

$\mathrm{P}=13.3-18.6 \mu \mathrm{m}, \mathrm{E}=13.3-18.6 \mu \mathrm{m}$.

Colpi long, narrow, 1.3-2.7 $\mu \mathrm{m}$ wide, with acute ends.

Endoapertures mainly indistinct, circular, 2.4-2.7 $\mu \mathrm{m}$ long and wide.

Exine $0.7-1.6 \mu \mathrm{m}$. Lindenbergia philippensis, L. sinaica.

Type II - 3-colpate

Subtype 1. Sculpture retipilate. Caput of pila $0.17-$ $0.33 \mu \mathrm{m}$.

$\mathrm{P}=38.6-47.9 \mu \mathrm{m}, \mathrm{E}=34.6-43.9 \mu \mathrm{m}$.

Colpi long, medium-width, $2.4-3.3 \mu \mathrm{m}$ wide, ends acute.

Exine 1.3-3.3 $\mu \mathrm{m}$. Bungea trifida.

Subtype 2. Sculpture retipilate and rugulateretipilate. Caput of pila $0.41-0.67 \mu \mathrm{m}$.

$\mathrm{P}=37.2-47.9 \mu \mathrm{m}, \mathrm{E}=33.2-42.6 \mu \mathrm{m}$.

Colpi medium-length, wide, $4.0-6.6 \mu \mathrm{m}$ wide, ends blunt, sometimes acute.

Exine 1.6-2.7 $\mu \mathrm{m}$. Cymbaria dahurica.

Subtype 3. Sculpture retipilate. Caput of pila 0.17$0.25 \mu \mathrm{m}$.

$\mathrm{P}=29.3-33.2 \mu \mathrm{m}, \mathrm{E}=30.6-35.9 \mu \mathrm{m}$.

Colpi medium-length, medium-width, $2.7-5.3 \mu \mathrm{m}$ wide, ends blunt, indistinct.

Exine 0.7-1.3 $\mu \mathrm{m}$. Cymbochasma borysthenica.

Subtype 4. Sculpture retipilate. Caput of pila 0.16$0.25 \mu \mathrm{m}$.

$\mathrm{P}=22.6-29.3 \mu \mathrm{m}, \mathrm{E}=25.3-29.3 \mu \mathrm{m}$.

Colpi long, narrow, 1.3-2.4 $\mu \mathrm{m}$ wide, ends blunt.

Exine 1.3-2.4 $\mu \mathrm{m}$. Siphonostegia chinensis.

\section{Descriptions of pollen grains}

Genus Lindenbergia Lehm.

Lindenbergia philippensis (Cham.) Benth. (Fig. 1, $a-c$; Fig. 4, $a-d$ )

LM. Pollen grains 3-colporate, oblate-spheroidal and spheroidal, occasionally prolate, in polar view 3-lobate, in equatorial view elliptical and circular. $\mathrm{P}=$ 13.3-17.3 $\mu \mathrm{m}, \mathrm{E}=14.6-18.6 \mu \mathrm{m}$. Colpi long, narrow, $1.3-2.7 \mu \mathrm{m}$ wide, with indistinct (some distinct) more or less strict margins, tapering to acute ends; colpus membrane smooth. Endoapertures indistinct, circular, 2.4-2.7 $\mu \mathrm{m}$ wide, 2.4-2.7 $\mu \mathrm{m}$ long. Mesocolpium = 9.3-10.6 $\mu \mathrm{m}$, apocolpium $=2.7-4.0(5.3) \mu \mathrm{m}$. Exine $1.1-1.6 \mu \mathrm{m}$ thick. Tectum nearly equal to infratectum. Columellae indistinct or distinct, thin. Exine sculpture indistinct or distinct, microreticulate. 
SEM. Sculpture reticulate. Colpus membrane smooth.

Specimen investigated: Philippines; Mountain Province; Bontoc Municipality; Bontoc territory; Caluttit; $17^{\circ} 05^{\prime} \mathrm{N}, 120^{\circ} 58^{\prime} \mathrm{E}$; $1000 \mathrm{~m}$ elev. 17 November 1982. C.C. Bodner. No 131 (MO).

Lindenbergia sinaica Benth. (Fig. 1, $d-f$; Fig. 4, $e-h$ )

LM. Pollen grains 3-colporate, prolate and spheroidal, occasionally oblate-spheroidal, in polar view 3-lobate, in equatorial view elliptical and circular. $\mathrm{P}=14.6-18.6 \mu \mathrm{m}, \mathrm{E}=13.3-17.3 \mu \mathrm{m}$. Colpi long, narrow, 2.0-2.4 $\mu \mathrm{m}$ wide, with indistinct (occasionally some distinct) more or less strict margins, tapering to acute ends; colpus membrane smooth. Endoapertures indistinct, more or less circular, $2.7 \mu \mathrm{m}$ wide, 2.4$2.7 \mu \mathrm{m}$ long. Mesocolpium $=7.9-10.6 \mu \mathrm{m}$, apocolpium $=4.0 \mu \mathrm{m}$. Exine $0.7-1.1 \mu \mathrm{m}$ thick, exine layers invisible. Exine sculpture indistinct, foveolate.

SEM. Sculpture reticulate. Colpus membrane smooth.

Specimen investigated: [Ethiopia]. About $150 \mathrm{~km}$. SW of Assab [Eritrea], along road to Kombolcha [Ethiopia]. Alt.: 400 m. Dry, rocky slope. 18.I 1966. W. de Wilde. No 9771 (MO).

Genus Bungea C.A. Mey.

Bungea trifida (Vahl) C.A. Mey. (Fig. 2, $a-c$; Fig. 5, $a-d)$

LM. Pollen grains 3-colpate, prolate and occasionally spheroidal, in polar view sub-triangular and/or circular, in equatorial view elliptical and circular. $\mathrm{P}=38.6-$ $47.9 \mu \mathrm{m}, \mathrm{E}=34.6-43.9 \mu \mathrm{m}$. Colpi long, medium-width, 2.4-3.3 $\mu \mathrm{m}$ wide, with indistinct, uneven margins, tapering to more or less acute or indistinct ends; colpus membrane granulate. Mesocolpium $=26.6-34.6 \mu \mathrm{m}$, apocolpium $=6.6-13.3 \mu \mathrm{m}$. Exine $1.3-3.3 \mu \mathrm{m}$ thick. Tectum nearly equal to infratectum. Columellae invisible. Exine sculpture indistinct, microreticulate.

SEM. Sculpture retipilate. Caput of pila 0.17$0.33 \mu \mathrm{m}$. Colpus membrane granulate.

Specimens investigated: 1. Armenian SSR [now Armenia], Vedi District [now in Ararat Province], right slope of the Vedi River, between Azizkend and Daynaz villages, phrygana. 27.V 1960. A. Takhtajan, E. Gabrielian, L.I. Mulkijanian (KW) [Label in Russian: Арм. ССР, Вединский р-н, правый борт р. Веди, c.c. Азизкенд $\times$ Дайназ, фригана. 27.V 1960. А. Тахтаджян, Э. Габриэлян, Л.И. Мулкиджанян]. 2. Turkey. B 10 Kars: Pamuk Dağ, 20 km from Iğdir to
Doğubayazit. 1600 m. Pastures. Perennial. Flower flava [sic!]. 30 May 1966. Davis, No 43867 (KW).

\section{Genus Cymbaria L.}

Cymbaria dahurica L. (Fig. 2, $d-f$; Fig. 5, $e-h$ )

LM. Pollen grains 3-colpate, prolate and occasionally oblate-spheroidal, in polar view sub-triangular, in equatorial view elliptical. $\mathrm{P}=37.2-47.9 \mu \mathrm{m}, \mathrm{E}=33.2$ $42.6 \mu \mathrm{m}$. Colpi medium-length, 4.0-6.6 $\mu \mathrm{m}$ wide, with indistinct, uneven margins, tapering to blunt, sometimes acute ends, colpus membrane smooth and granulate. Mesocolpium $=26.6-33.2 \mu \mathrm{m}$, apocolpium $=10.6-$ $19.9 \mu \mathrm{m}$. Exine $1.6-2.7 \mu \mathrm{m}$ thick. Tectum nearly equal to infratectum. Columellae indistinct. Exine sculpture indistinct, sometimes distinct, microreticulate.

SEM. Sculpture retipilate and rugulate-retipilate. Caput of pila $0.41-0.67 \mu \mathrm{m}$. Colpus membrane granulate.

Specimens investigated: 1 . Khakassian Autonomous Region [now Republic of Khakassia, Russian Federation], Altai District, near Ochury village. Southern rocky slope, fine-turfgrass steppe. 30 June 1969. A. Koroleva (KW) [Label in Russian: Хакасская авт. обл., Алтайский р-н, окр. с. Очуры. Южный каменистый склон, мелко-дерновинная степь. 30.VI 1969. А. Королева]. 2. Transbaikalia [now Zabaykalsky Krai, Russian Federation]. On dry mountain slopes and in steppes near Nerchinsk, abundant. June 1898. Coll. Gubelman and Migunov (KW) [Label in Russian: Забайкалье. На сухих склонах гор и в степях около Нерчинска в изобилии. Июнь 1898; Собр. Губельман и Мигунов].

\section{Genus Cymbochasma (Endl.) Klokov \& Zoz}

Cymbochasma borysthenica (Pall. ex Schlecht.) Klokov \& Zoz (Cymbaria borysthenica Pall. ex Schlecht.) (Fig. 3, $a-c$; Fig. 5, $i-l$ )

LM. Pollen grains 3-colpate, occasionally 4-colpate, oblate-spheroidal, in polar view sub-circular, in equatorial view elliptical. $\mathrm{P}=29.3-33.2 \mu \mathrm{m}, \mathrm{E}=30.6-$ $35.9 \mu \mathrm{m}$. Colpi medium-length, medium-width, 2.7$5.3 \mu \mathrm{m}$ wide, with indistinct, uneven margins, tapering to blunt, indistinct ends, colpus membrane smooth and granulate. Mesocolpium $=22.6-26.6 \mu \mathrm{m}$, apocolpium $=6.6-9.3 \mu \mathrm{m}$. Exine $0.7-1.3 \mu \mathrm{m}$ thick. Tectum nearly equal to infratectum. Columellae indistinct. Exine sculpture indistinct, sometimes distinct, microreticulate.

SEM. Sculpture retipilate. Caput of pila $0.17-$ $0.25 \mu \mathrm{m}$. Colpus membrane granulate. 


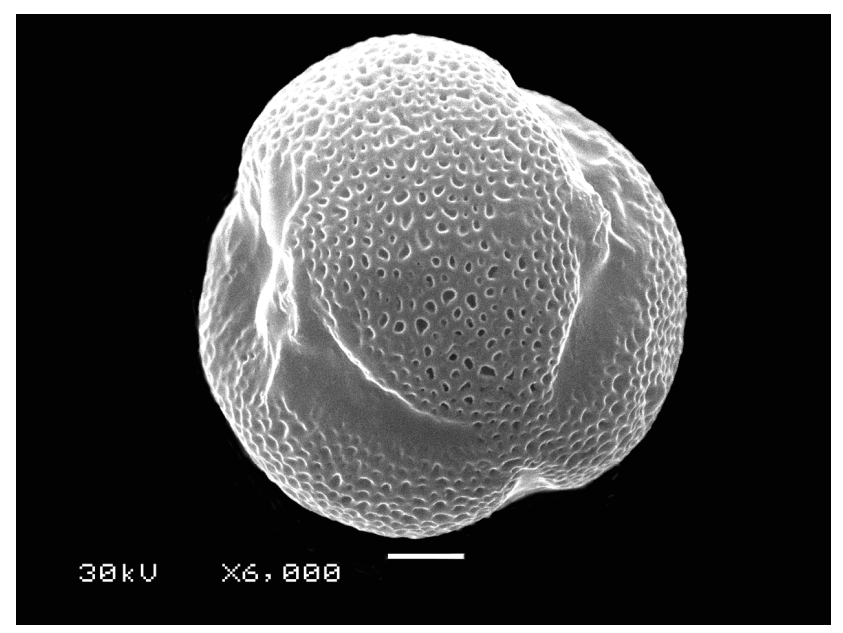

a

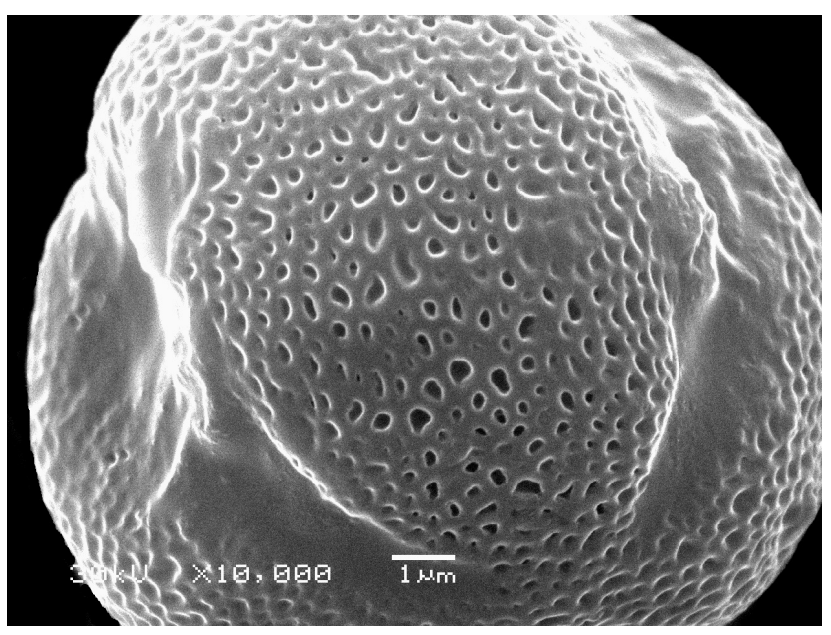

c

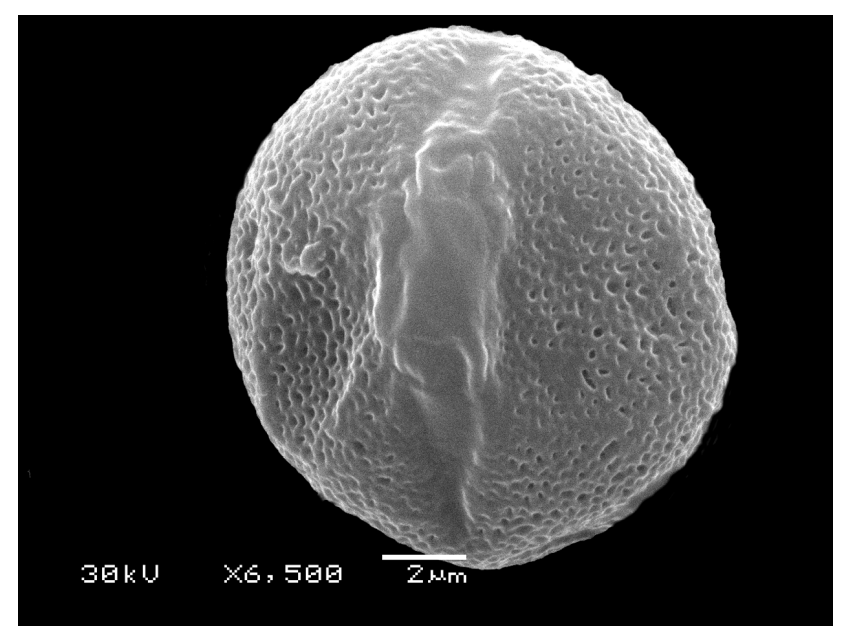

$\boldsymbol{e}$

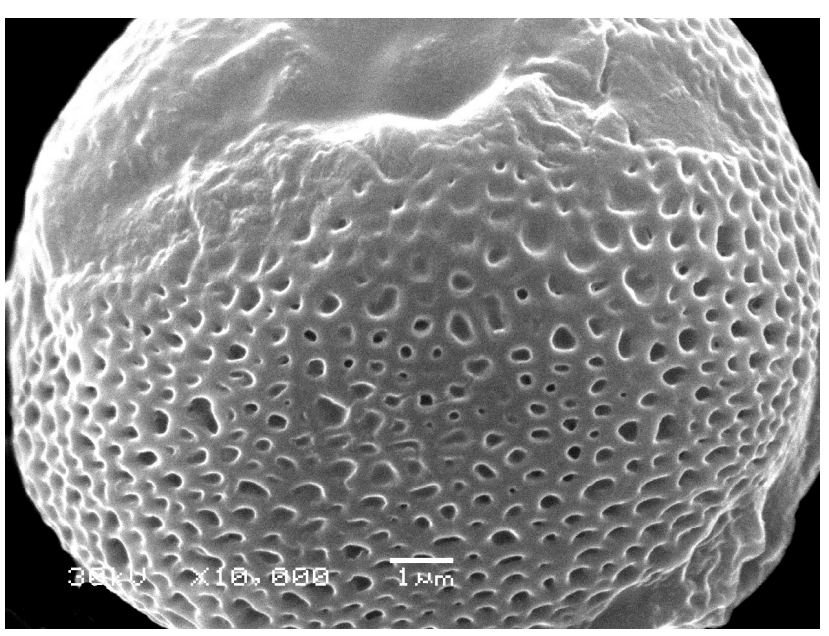

b
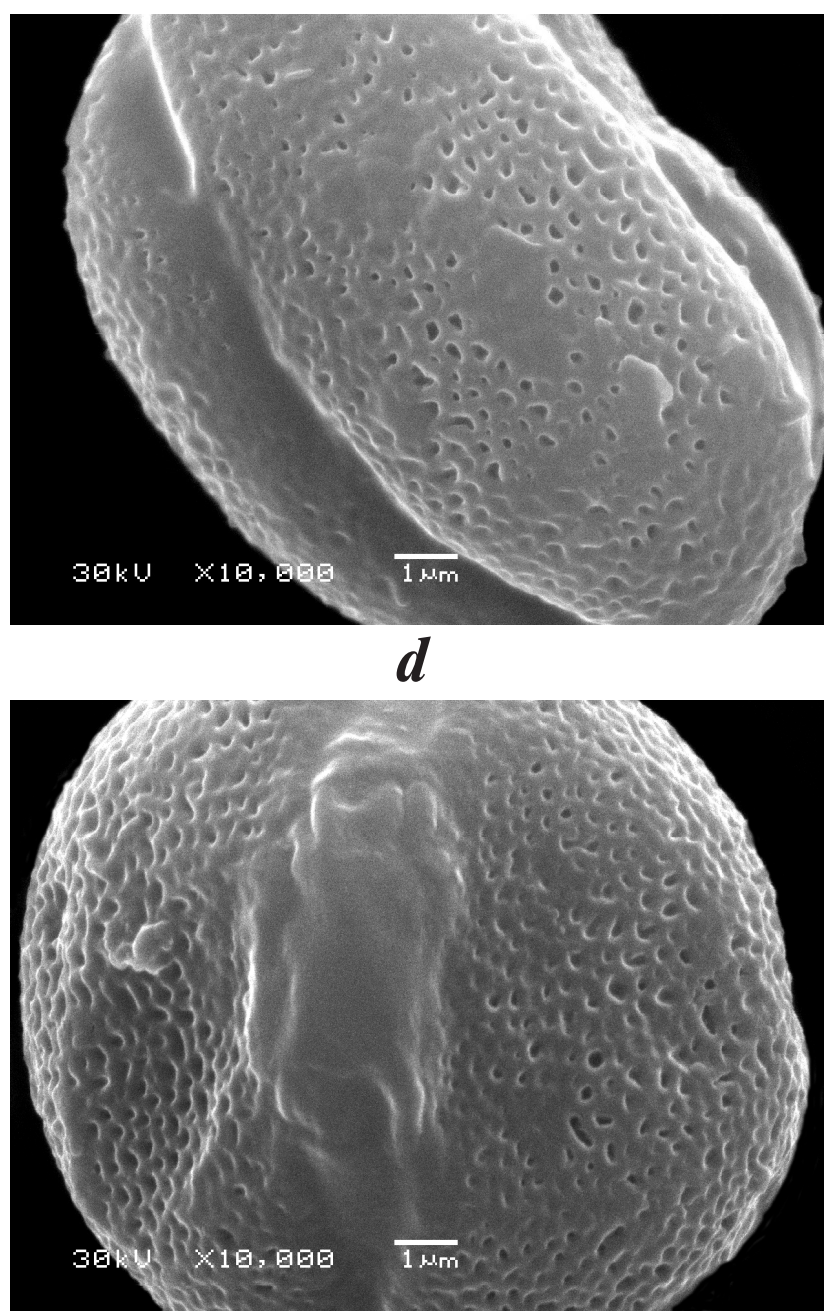

$f$

Fig. 1. Pollen grains of Lindenbergia (scanning electron microscopy): $a-c-L$. philippensis; $d-f-L$. sinaica; $a-$ polar and equatorial view; $e-$ equatorial view; $b-d, f-$ reticulate exine sculpture 


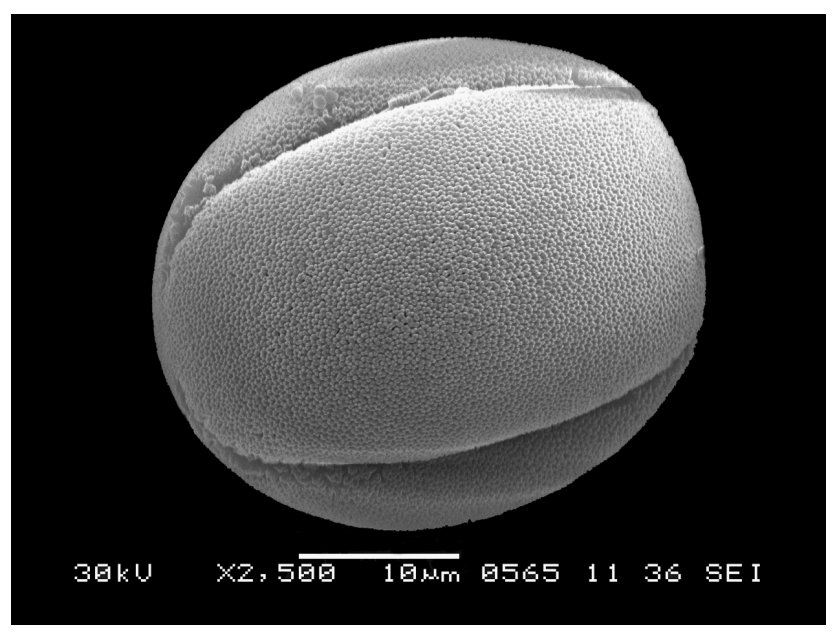

a

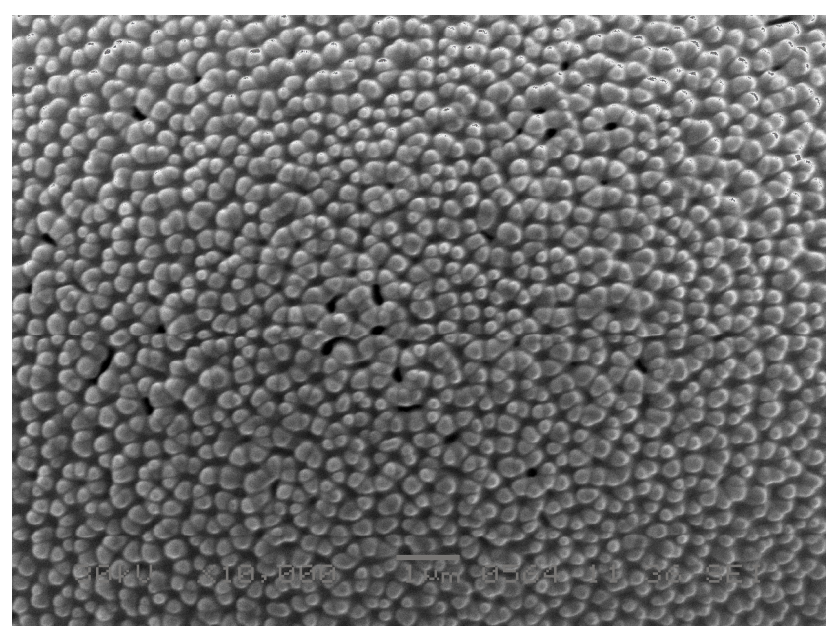

c

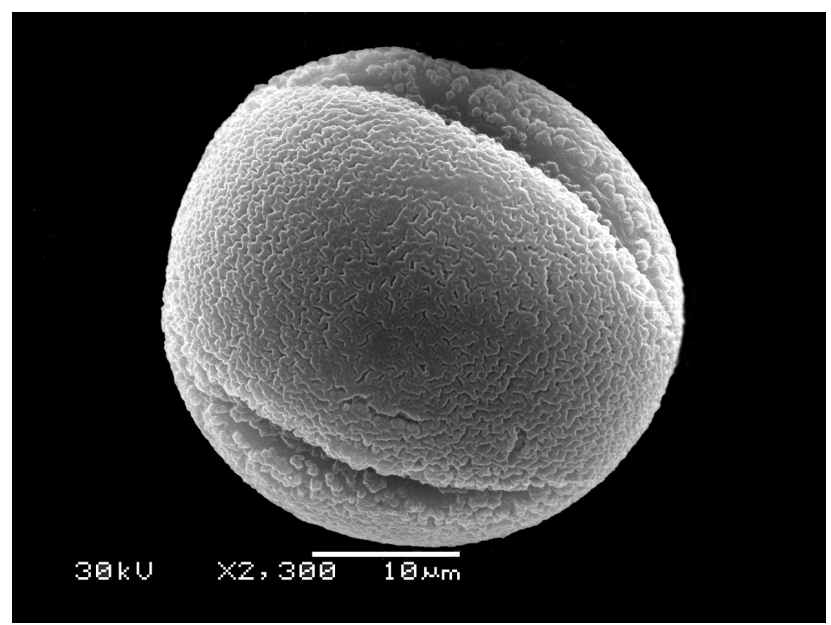

$\boldsymbol{e}$

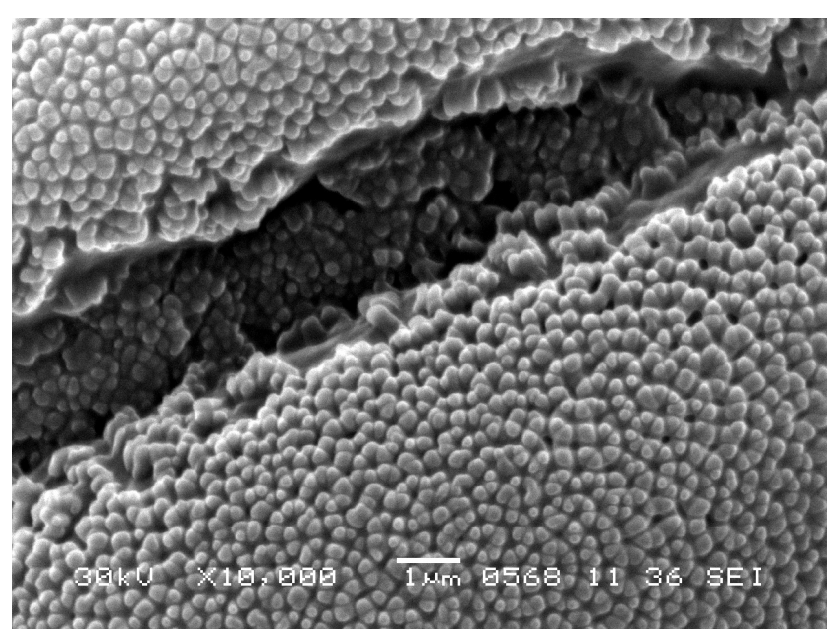

b

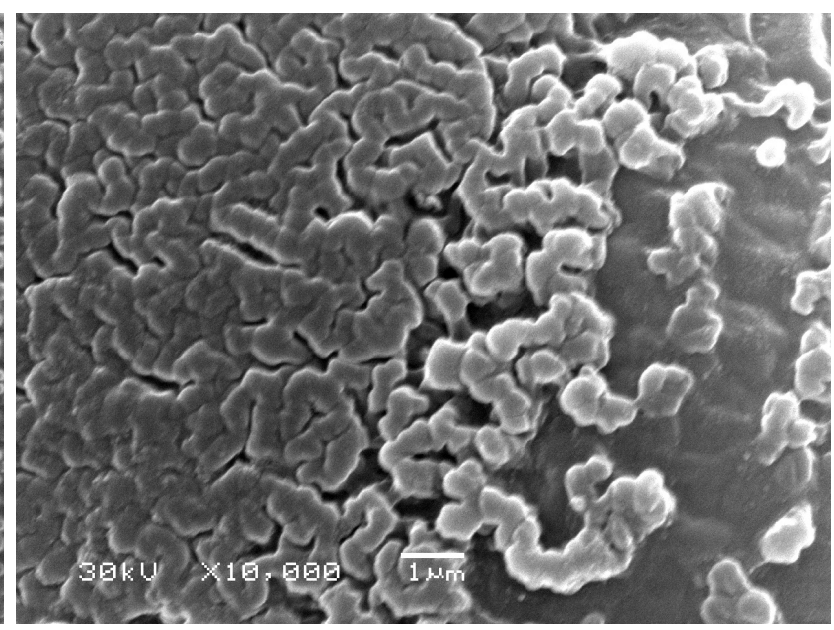

d

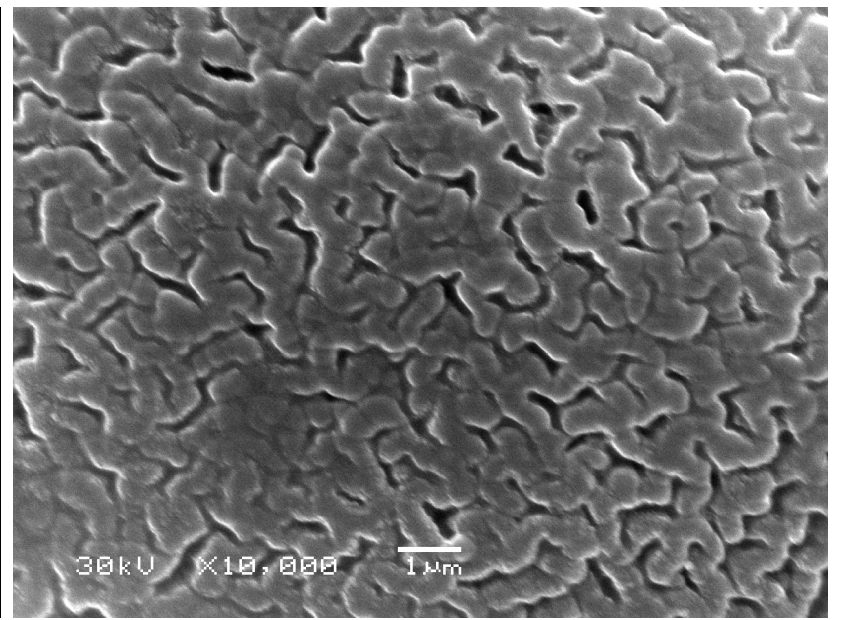

$f$

Fig. 2. Pollen grains of Bungea and Cymbaria (scanning electron microscopy): $a-c-B$. trifida; $d-f-C$. dahurica; $a, e-$ equatorial view; $b, c$ - retipilate exine sculpture; $d, f$ - rugulate-retipilate exine sculpture 


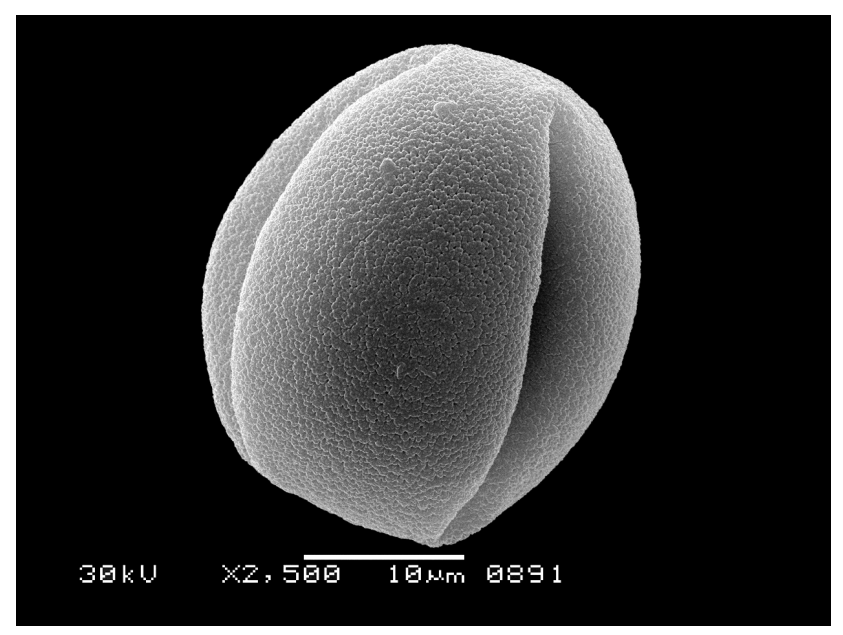

$\boldsymbol{a}$

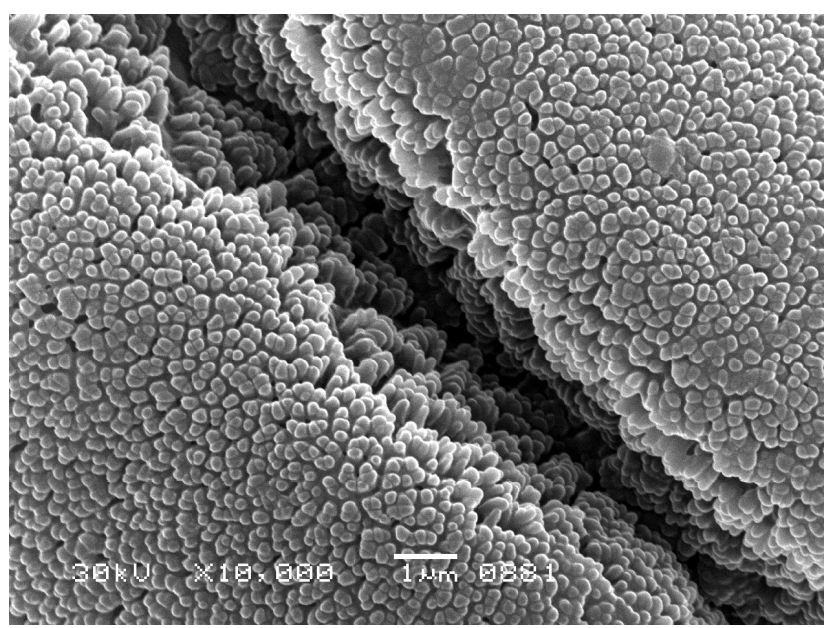

\section{c}

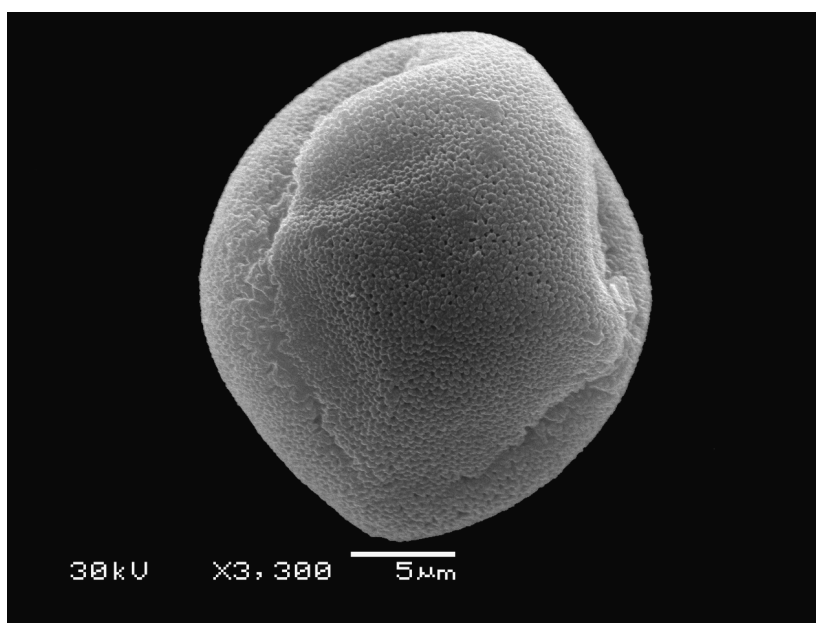

$\boldsymbol{e}$

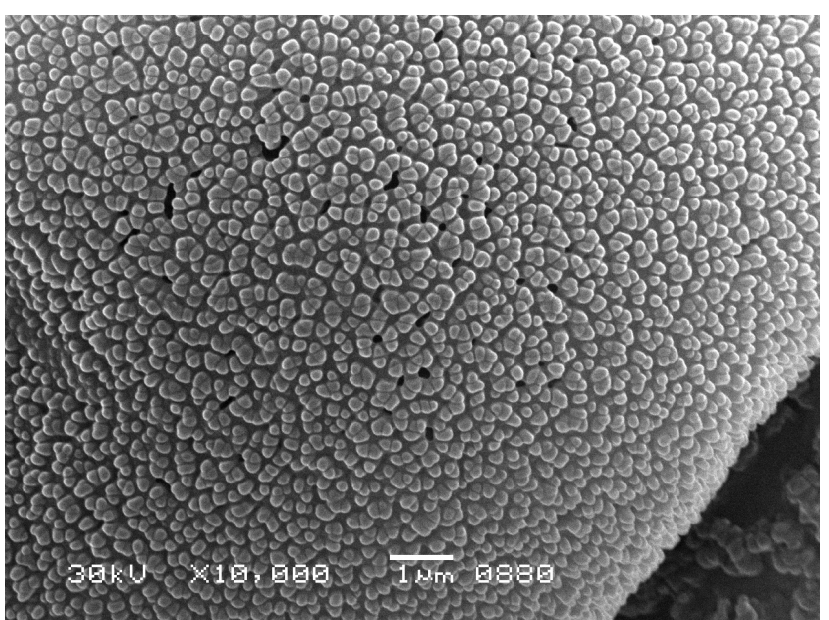

b

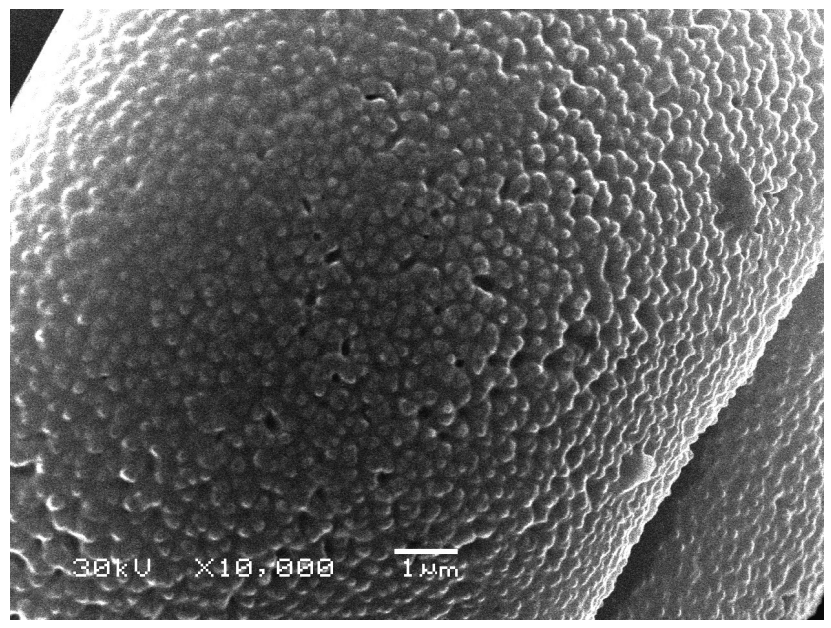

d
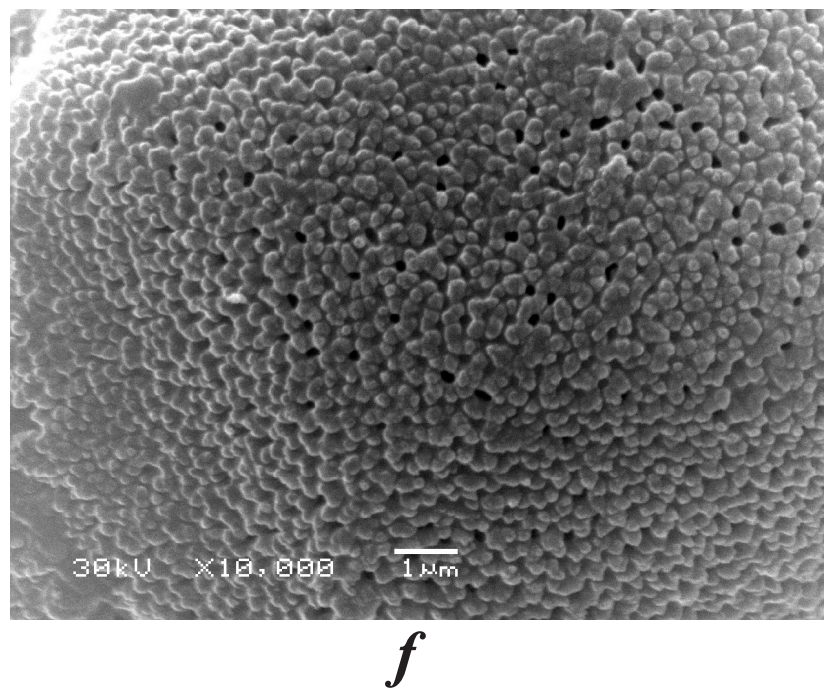

Fig. 3. Pollen grains of Cymbochasma and Siphonostegia (scanning electron microscopy): $a-c-C$. borysthenica; $d-f-$ S. chinensis; $a, e$ - equatorial view; $b-d, f$ - retipilate exine sculpture 


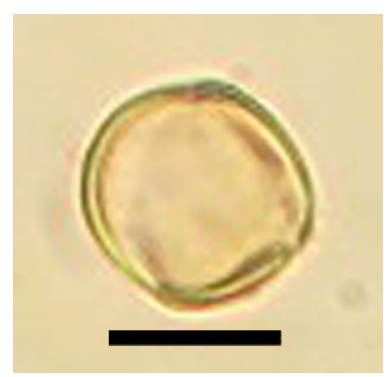

$\boldsymbol{a}$

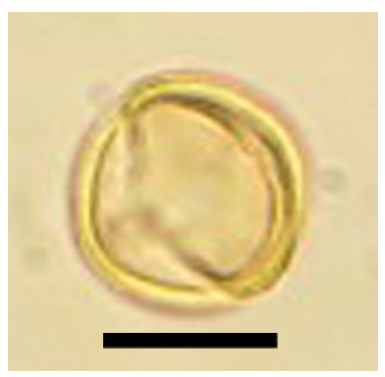

$\boldsymbol{e}$

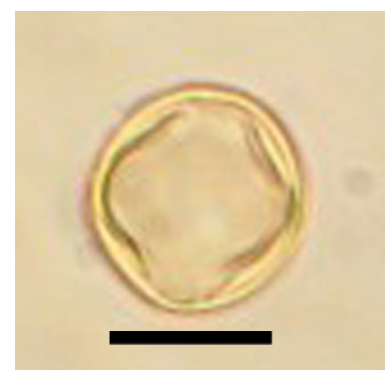

b

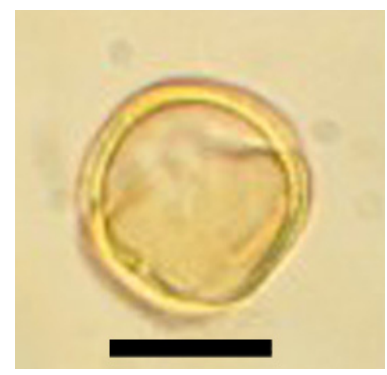

$f$

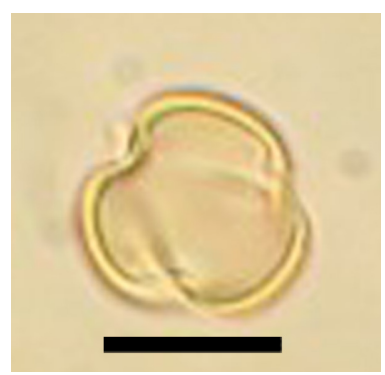

c

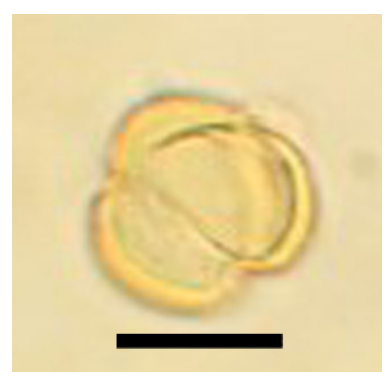

g

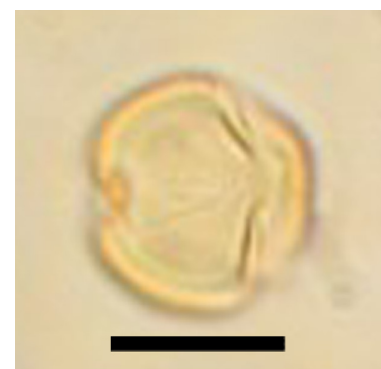

$d$

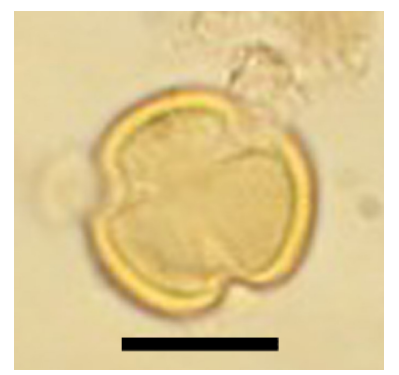

$\boldsymbol{h}$

Fig. 4. Pollen grains of Lindenbergia (light microscopy): $a-d-L$. philippensis; $e-h-L$. sinaica; $a, b, e, f-$ equatorial view; $c, d, \mathrm{~g}, h-$ polar view. Scale bars: $10 \mu \mathrm{m}$

Specimens investigated: 1. Ukrainian SSR [now Ukraine], Kherson Region, Kakhovka District, between Lvovo [L'vove] and Kozatskoye [Kozats'ke] villages, slopes of a large ravine of western exposition, petrophilic steppe area, on limestone. 24 April 1972. B.V. Zaverukha, No. 058169 (KW) [Label in Russian: УССР, Херсонская обл., Каховский р-н, между селами Львово - Козацкое, склоны большой балки западной экспозиции, степной участок петрофильного х-ра, на известняках. 24.IV 1972. Б.В. Заверуха. № 058169]. 2. [Ukraine] Mykolayiv Region, Snigurivka town, limestone slopes at the Ingulets River. 20 April 1972. L. Krytzka, No. 040211 (KW) [Label in Ukrainian: Миколаївська обл., м. Снігурівка, вапнякові схили на р. Інгулець. 20.IV 1972. Л. Крицька. № 040211].

\section{Genus Siphonostegia Benth.}

Siphonostegia chinensis Benth. (Fig. 3, $d-f$; Fig. 5, $m-p)$

LM. Pollen grains 3-colpate, oblate-spheroidal, spheroidal, occasionally prolate, in polar view circular, in equatorial view circular. $\mathrm{P}=22.6-29.3 \mu \mathrm{m}, \mathrm{E}=$ 25.3-29.3 $\mu \mathrm{m}$. Colpi long, narrow, $1.3-2.4 \mu \mathrm{m}$ wide, with indistinct, uneven margins, tapering to blunt ends, colpus membrane granulate. Mesocolpium = 18.6-
$22.6 \mu \mathrm{m}$, apocolpium $=5.3-9.3 \mu \mathrm{m}$. Exine $1.3-2.4 \mu \mathrm{m}$ thick. Tectum nearly equal to infratectum. Columellae distinct, thin, arranged more or less regularly. Exine sculpture distinct, microreticulate.

SEM. Sculpture retipilate. Caput of pila 0.16$0.25 \mu \mathrm{m}$. Colpus membrane granulate.

Specimen investigated: [Russia, Russian Far East] Primorye Province, near Khabarovsk city, in sands. 11 July 1910. N. Desoulavi (KW) [Label in Latin and Russian: Herbarium Florae Rossicae. Prov. Primorskaja, pr. urb. Chabarowsk, in arenosis. 11.VII 1910. Н. Десулави].

Original and published data on quantitative and qualitative pollen characters of representative of Cymbarieae and Lindenbergieae are summarized in Tables 1 and 2.

Comparative pollen morphology of genera of Cymbarieae and Lindenbergieae

In general, our data are in good agreement with the results of previous studies (Inceoğlu, 1982; Minkin, Eshbaugh, 1989; Lu et al., 2007). Analysis of our original palynomorphological data and literature records demonstrated that pollen grains of representatives of all genera of Cymbarieae are characterized by the 3-colpate type of apertures, mainly with retipilate sculpture (Table 2). 


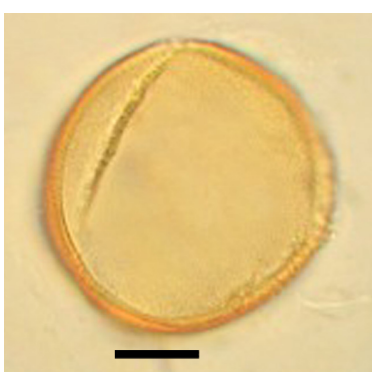

$a$
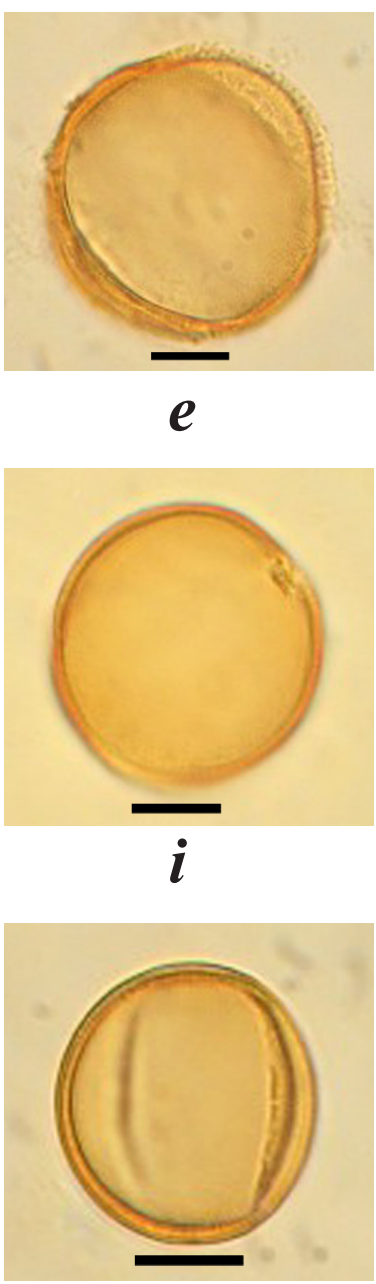

m

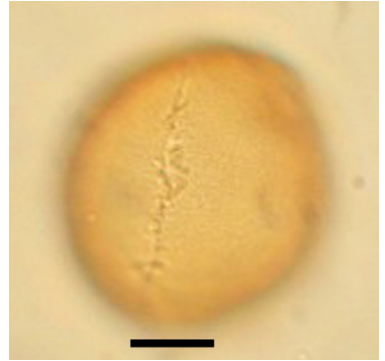

b
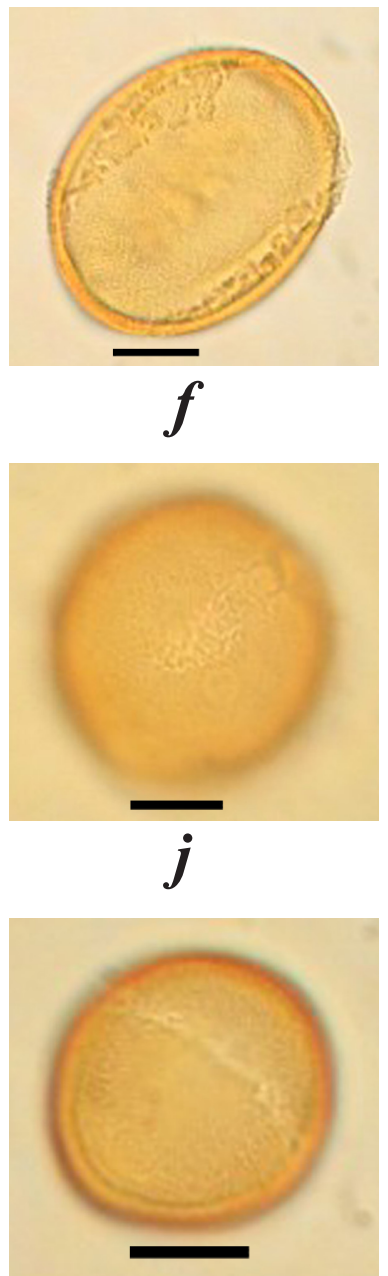

n

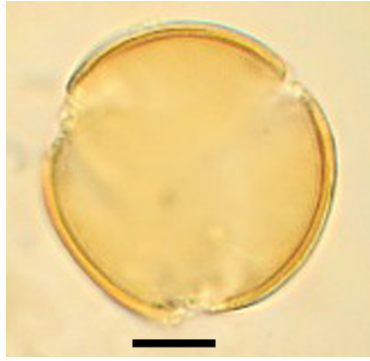

c

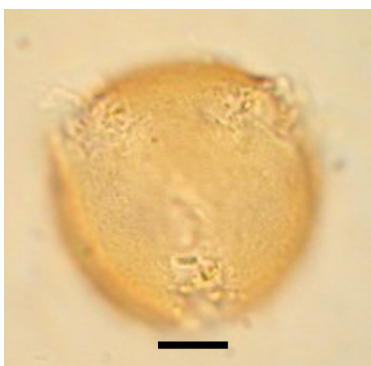

g
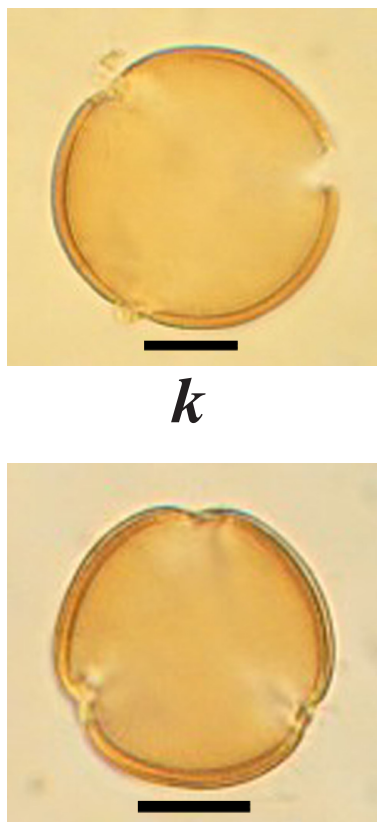

O
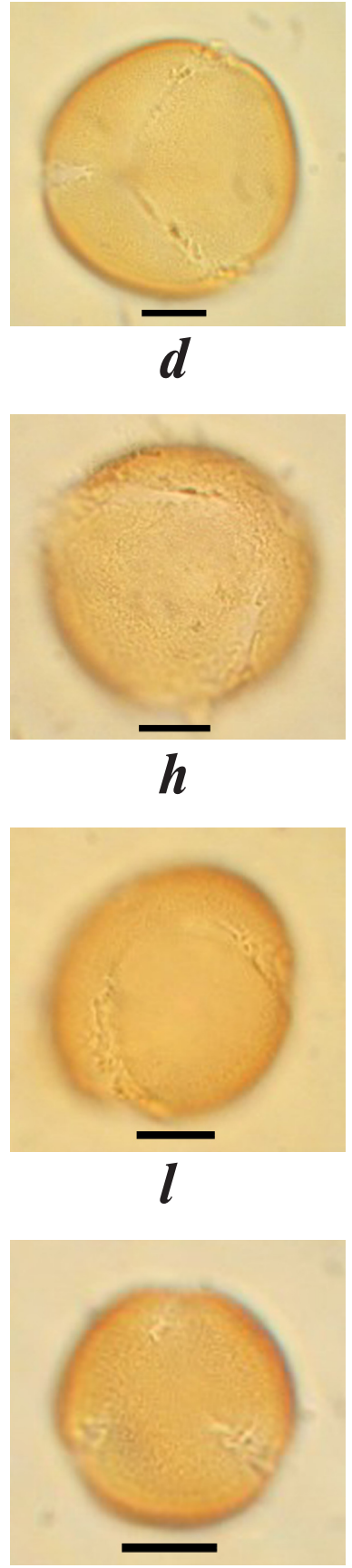

p

Fig. 5. Pollen grains of Bungea, Cymbaria, Cymbochasma, and Siphonostegia (light microscopy): $a-d-B$. trifida; $e-h-$ $C$. dahurica; $i-l-C$. borysthenica; $m-p-S$. chinensis. $a, b, e, f, i, j, m, n-$ equatorial view; $c, d, g, h, k, l, o, p-$ polar view. Scale bars: $10 \mu \mathrm{m}$. 
Table 1. Summary of pollen morphometric features

\begin{tabular}{|l|c|c|c|c|c|c|c|}
\hline \multicolumn{1}{|c|}{ Taxon } & $\begin{array}{c}\text { Polar axis } \\
(\mu \mathrm{m})\end{array}$ & $\begin{array}{c}\text { Equatorial axis } \\
(\mu \mathrm{m})\end{array}$ & $\begin{array}{c}\text { Colpi width } \\
(\mu \mathrm{m})\end{array}$ & $\begin{array}{c}\text { Mesocolpium } \\
(\mu \mathrm{m})\end{array}$ & $\begin{array}{c}\text { Apocolpium } \\
(\mu \mathrm{m})\end{array}$ & Exine $(\mu \mathrm{m})$ & Reference \\
\hline Lindenbergia philippensis & $13.3-17.3$ & $14.6-18.6$ & $1.3-2.7$ & $9.3-10.6$ & $2.7-4.0(5.3)$ & $1.1-1.6$ & original data \\
\hline Lindenbergia sinaica & $14.6-18.6$ & $13.3-17.3$ & $2.0-2.4$ & $7.9-10.6$ & $3.3-4.0$ & $0.7-1.1$ & original data \\
\hline Bungea trifida & $38.6-47.9$ & $34.6-43.9$ & $2.4-3.3$ & $26.6-34.6$ & $6.6-13.3$ & $1.3-3.3$ & original data \\
\hline Bungea trifida & 35 & 39 & - & - & 7 & 1.1 & Inceoğlu, 1982 \\
\hline Cymbaria dahurica & $37.2-47.9$ & $33.2-42.6$ & $4.0-6.6$ & $26.6-33.2$ & $10.6-19.9$ & $1.6-2.7$ & original data \\
\hline Cymbaria dahurica & $32.5-42.5$ & $27.5-35.0$ & - & - & - & - & Lu et al., 2007 \\
\hline Cymbaria mongolica & $37.5-50.0$ & $30.0-40.0$ & - & - & - & - & Lu et al., 2007 \\
\hline Cymbochasma borysthenica & $29.3-33.2$ & $30.6-35.9$ & $2.7-5.3$ & $22.6-26.6$ & $6.6-9.3$ & $0.7-1.3$ & original data \\
\hline Monochasma savatieri & $32.5-43.8$ & $27.5-41.3$ & - & - & - & - & Lu et al., 2007 \\
\hline Siphonostegia chinensis & $22.6-29.3$ & $25.3-29.3$ & $1.3-2.4$ & $18.6-22.6$ & $5.3-9.3$ & $1.3-2.4$ & original data \\
\hline Siphonostegia chinensis & 22.1 & 22.4 & - & - & - & - & Minkin, Eshbaugh, 1989 \\
\hline Siphonostegia chinensis & $22.5-30.0$ & $23.8-32.5$ & - & - & - & - & Lu et al., 2007 \\
\hline $\begin{array}{l}\text { Siphonostegia syriaca } \\
\text { (Lesquereuxia syriaca })\end{array}$ & 24 & 24 & - & - & 11 & 0.8 & Inceoğlu, 1982 \\
\hline Schwalbea americana & 27.2 & 27.2 & - & - & - & - & Minkin, Eshbaugh, 1989 \\
\hline
\end{tabular}

Original data are those reported here and in Table 2; "-" means no data reported.

Table 2. Summary of pollen morphological characteristics

\begin{tabular}{|c|c|c|c|c|c|c|c|}
\hline Taxon & Apertures & Shape & Polar view & Colpi & $\begin{array}{l}\text { Colpus } \\
\text { membrane }\end{array}$ & $\begin{array}{l}\text { Exine sculpture/ } \\
\text { caput of pila }\end{array}$ & Reference \\
\hline $\begin{array}{l}\text { Lindenbergia } \\
\text { philippensis }\end{array}$ & 3-colporate & $\begin{array}{c}\text { oblate-spheroidal, } \\
\text { spheroidal (some prolate) }\end{array}$ & trilobate & $\begin{array}{l}\text { long, narrow, } \\
\text { acute ends }\end{array}$ & smooth & reticulate & $\begin{array}{l}\text { original } \\
\text { data }\end{array}$ \\
\hline $\begin{array}{l}\text { Lindenbergia } \\
\text { sinaica }\end{array}$ & 3-colporate & $\begin{array}{l}\text { prolate, spheroidal (some } \\
\text { oblate-spheroidal) }\end{array}$ & trilobate & $\begin{array}{l}\text { long, narrow, } \\
\text { acute ends }\end{array}$ & smooth & reticulate & $\begin{array}{l}\text { original } \\
\text { data }\end{array}$ \\
\hline Bungea trifida & 3-colpate & prolate (some spheroidal) & $\begin{array}{c}\text { sub-triangular, } \\
\text { circular }\end{array}$ & $\begin{array}{l}\text { long, medium-width, } \\
\text { acute ends }\end{array}$ & granulate & $\begin{array}{l}\text { retipilate, } \\
0.17-0.33 \\
\end{array}$ & $\begin{array}{l}\text { original } \\
\text { data }\end{array}$ \\
\hline Bungea trifida & 3-colpate & oblate-spheroidal & semi-angular & long, acute ends & $\begin{array}{l}\text { coarsely } \\
\text { granulate }\end{array}$ & retipilate & $\begin{array}{l}\text { Inceoğlu, } \\
1982\end{array}$ \\
\hline Cymbaria dahurica & 3-colpate & $\begin{array}{l}\text { prolate (some oblate- } \\
\text { spheroidal) }\end{array}$ & sub-triangular & $\begin{array}{l}\text { medium-length, } \\
\text { wide, blunt or acute } \\
\text { ends }\end{array}$ & granulate & $\begin{array}{c}\text { retipilate, } \\
\text { rugulate-retipilate, } \\
0.41-0.67\end{array}$ & $\begin{array}{l}\text { original } \\
\text { data }\end{array}$ \\
\hline Cymbaria dahurica & 3-colpate & subprolate & sub-triangular & $\begin{array}{l}\text { wide at equator and } \\
\text { narrow near poles }\end{array}$ & $\begin{array}{l}\text { with } \\
\text { granules }\end{array}$ & $\begin{array}{l}\text { retipilate, } \\
0.45-0.90\end{array}$ & $\begin{array}{l}\text { Lu et al., } \\
2007\end{array}$ \\
\hline Cymbaria mongolica & 3-colpate & subprolate & sub-triangular & $\begin{array}{l}\text { narrow, long and } \\
\text { sunken }\end{array}$ & $\begin{array}{c}\text { with } \\
\text { granules }\end{array}$ & $\begin{array}{l}\text { retipilate, } \\
0.16-0.40\end{array}$ & $\begin{array}{l}\text { Lu et al., } \\
2007\end{array}$ \\
\hline $\begin{array}{l}\text { Cymbochasma } \\
\text { borysthenica }\end{array}$ & $\begin{array}{l}\text { 3-colpate } \\
\text { (some } \\
\text { 4-colpate) } \\
\end{array}$ & oblate-spheroidal & sub-circular & $\begin{array}{c}\text { medium-length, } \\
\text { medium-width, } \\
\text { blunt, indistinct ends }\end{array}$ & granulate & $\begin{array}{l}\text { retipilate, } \\
0.17-0.25\end{array}$ & $\begin{array}{l}\text { original } \\
\text { data }\end{array}$ \\
\hline $\begin{array}{l}\text { Monochasma } \\
\text { savatieri }\end{array}$ & 3-colpate & $\begin{array}{c}\text { prolate, spheroidal (some } \\
\text { oblate-spheroidal) }\end{array}$ & circular & $\begin{array}{l}\text { wide at equator and } \\
\text { narrow near poles }\end{array}$ & $\begin{array}{l}\text { with fine } \\
\text { granules }\end{array}$ & $\begin{array}{l}\text { retipilate, } \\
0.19-0.39 \\
\end{array}$ & $\begin{array}{l}\text { Lu et al., } \\
2007\end{array}$ \\
\hline $\begin{array}{l}\text { Siphonostegia } \\
\text { chinensis }\end{array}$ & 3-colpate & $\begin{array}{c}\text { spheroidal, oblate- } \\
\text { spheroidal (some prolate) }\end{array}$ & circular & $\begin{array}{c}\text { long, narrow, blunt } \\
\text { ends }\end{array}$ & granulate & $\begin{array}{l}\text { retipilate, } \\
0.16-0.25\end{array}$ & $\begin{array}{c}\text { original } \\
\text { data }\end{array}$ \\
\hline $\begin{array}{l}\text { Siphonostegia } \\
\text { chinensis }\end{array}$ & 3-colpate & spheroidal & - & - & - & retipilate & $\begin{array}{c}\text { Minkin, } \\
\text { Eshbaugh, } \\
1989\end{array}$ \\
\hline $\begin{array}{l}\text { Siphonostegia } \\
\text { chinensis }\end{array}$ & 3-colpate & spheroidal & circular & $\begin{array}{c}\text { wide at equator, but } \\
\text { not narrow near poles }\end{array}$ & $\begin{array}{l}\text { with coarse } \\
\text { granules }\end{array}$ & $\begin{array}{l}\text { retipilate, } \\
0.18-0.31\end{array}$ & $\begin{array}{l}\text { Lu et al., } \\
2007\end{array}$ \\
\hline $\begin{array}{l}\text { Siphonostegia syriaca } \\
\text { (Lesquereuxia } \\
\text { syriaca) }\end{array}$ & 3-colpate & prolate, spheroidal & circular & long, acute ends & $\begin{array}{l}\text { coarsely } \\
\text { granulate }\end{array}$ & retipilate & $\begin{array}{l}\text { Inceoğlu, } \\
1982\end{array}$ \\
\hline $\begin{array}{l}\text { Schwalbea } \\
\text { americana }\end{array}$ & 3-colpate & spheroidal & - & - & - & retipilate & $\begin{array}{c}\text { Minkin, } \\
\text { Eshbaugh, } \\
1989\end{array}$ \\
\hline
\end{tabular}


Pollen grains in Cymbochasma borysthenica are 3-colpate and occasionally 4-colpate (Tsymbalyuk, 2011). They have the thinnest exine $(0.7-1.3 \mu \mathrm{m})$ among pollen grains of the studied species of the tribe. Pollen grains of Cymbochasma borysthenica are smaller as compared to pollen of Cymbaria dahurica and C. mongolica, and also differ from species of Cymbaria sensu stricto in the pollen shape and outline (see Table 2). Thus, pollen characters may provide additional evidence in favor of recognition of Cymbochasma as a separate genus.

The two studied species of Cymbaria sensu stricto are similar in their pollen size, shape, and outline; however, they differ in their colpi structure and exine sculpture. In pollen grains of Cymbaria dahurica, colpi are medium-length, wider than in $C$. mongolica, and exine sculpture is retipilate (caput of pila $0.41-0.90 \mu \mathrm{m}$ ) or rugulate-retipilate, while $C$. mongolica has narrower and longer colpi (as compared to those in $C$. dahurica) and retipilate exine sculpture (caput of pila $0.16-0.40 \mu \mathrm{m}$ ) (Lu et al., 2007, and original data).

In general, pollen grains of Bungea trifida and Cymbaria mongolica (Lu et al., 2007) are similar in their shape, outline, size, and exine sculpture; however, Bungea trifida has colpi wider than those in C. mongolica, and the thickest exine $(1.3-3.3 \mu \mathrm{m})$ among all studied species.

The smallest sizes are characteristic of pollen grains of Siphonostegia chinensis (Table 1). This species also has the narrowest colpi among all studied species. Columellae in all species studied here are mainly indistinct, while in Siphonostegia chinensis those are distinct, thin, and arranged more or less regularly.

Pollen grains of Siphonostegia syriaca (Lesquereuxia syriaca) (Inceoğlu, 1982) and S. chinensis are similar in their shape, outline, size, exine sculpture, and length of colpi; however, in S. syriaca the colpi ends are acute, while in $S$. chinensis they are obtuse (with blunt ends).

Pollen grains of Schwalbea (Minkin, Eshbaugh, 1989) are similar to those of Siphonostegia in their outline, size, and exine sculpture. Unfortunately, the characters reported by Minkin and Eshbaugh (1989) are insufficient for a more detailed comparative analysis.

The two studied species of Lindenbergia are similar to each other in having the 3-colporate aperture type, reticulate exine sculpture, and long and narrow colpi. They, however, differ by the exine thickness: in L. philippensis the exine is 1.1-1.6 $\mu \mathrm{m}$ thick, the tectum is nearly equal to the infratectum, and columellae are indistinct or distinct, while in $L$. sinaica the exine is thinner $(0.7-1.1 \mu \mathrm{m}$ thick), and exine layers are invisible (indistinct).

Comparison of palynomorphological and molecular phylogenetic evidence

According to molecular phylogenetic studies (Bennett, Mathews, 2006; McNeal et al., 2013), the clade of Cymbarieae is subdivided into two subclades. The subclade that includes Bungea, Cymbaria, Cymbochasma, and Monochasma is characterized by larger pollen grains and wider colpi, as compared to the subclade of Siphonostegia (including Lesquereuxia) and Schwalbea.

The earliest-branching clade of Lindenbergia is sister to the clade containing all other members of Orobanchaceae. Small-sized 3-colporate pollen grains with reticulate exine revealed in all studied taxa of Lindenbergia are similar to pollen of some representatives of Plantaginaceae, in particular, those of Gratioleae (Tsymbalyuk, Mosyakin, 2013a, 2014; Tsymbalyuk, 2016). In our opinion, that superficial similarity does not reflect direct phylogenetic relationships of these taxa, but rather some recurrent patterns (plesiomorphic characters) appearing is several clades of Lamiales.

The genus Paulownia Siebold \& Zucc. is currently placed phylogenetically as a group sister to Orobanchaceae (Olmstead et al., 2001; Oxelman et al., 2005; Bennet, Mathews, 2006; Schäferhoff et al., 2010; McNeal et al., 2013). Pollen grains of Paulownia are 3-colporate, with reticulate exine (Erdtman, 1952; Chen, 1983; Tsymbalyuk, 2014). In these characters, Paulownia is rather similar to Lindenbergia. However, Paulownia differ from Lindenbergia in having small- and medium-sized pollen grains (small in Lindenbergia), trilobate and sub-triangular in outline (only trilobate in Lindenbergia), with distinct orae (indistinct in Lindenbergia) and the colpus membrane smooth and granulate (only smooth in Lindenbergia). Some similarity with pollen of Paulownia and Lindenbergia is also observed in pollen grains of the phylogenetically still problematic genus Wightia Wall. (Zhou et al. 2014), which also has 3-colporate pollen with reticulate exine sculpture (Wei, 1989; Tsymbalyuk, 2014, 2016). However, in Paulownia and Lindenbergia orae are circular and colpi are tapered to acute ends, while Wightia has eliptical orae and colpi expanded to rounded ends.

Thus, the earliest-branching clade of Orobanchaceae (Lindenbergieae) is palynomorphologically similar to the clade sister to all Orobanchaceae (Paulowniaceae) 
and to the currently phylogenetically unplaced (?) genus Wightia. These palynomorphological findings confirm the phylogenetic patterns currently revealed in basal Orobanchaceae and their closest relatives.

\section{Main trends of pollen evolution in early-branching Orobanchaceae}

We may conclude that the colporate (most probably 3-colporate) type of pollen grains could be ancestral in Orobanchaceae. This type is peculiar to members of Clade I (sensu McNeal et al., 2013) that includes Lindenbergia (3-colporate pollen with reticulate exine sculpture and indistinct orae). Then, the colpate type probably evolved by reduction of orae; this type, with retipilate exine sculpture, is characteristic of representatives of Clade II, including Siphonostegia, Schwalbea, Monochasma, Cymbaria, Cymbochasma, and Bungea. A sculpture type transitional between reticulate and retipilate exine could be the rugulate-retipilate type observed in Cymbaria dahurica. In the large and diverse Clade III, which includes holoparasitic members of Boschniakia C.A. Mey. ex Bong., Xylanche Beck, Kopsiopsis (Beck) Beck, Epifagus Nutt., Conopholis Wallr., Mannagettaea Harry Sm., Cistanche Hoffmanns. \& Link, Diphelypaea Nicolson, Orobanche L., Phelipanche Pomel, and the recently resurrected genus Aphyllon Mitch. (Schneider, 2016; Schneider, Moore, 2017), a greater diversity of aperture and exine sculpture types and patterns is observed (Tsymbalyuk, Mosyakin, 2013b, c; Zare et al., 2014; Piwowarczyk et al., 2015; Tsymbalyuk, 2016).

Thus, available palynomorphological data are well consistent with the phylogenetic patterns in early-branching Orobanchaceae (Lindenbergieae and Cymbarieae) and their relatives, which are currently revealed by molecular phylogenetic evidence (Bennett, Mathews, 2006; McNeal et al., 2013). The considerable pollen diversity in crown clades and subclades of Orobanchaceae evolved on the base of a few pollen types and subtypes, which were peculiar to hypothetical ancestors of Orobanchaceae and are probably preserved until now in the extant taxa of Paulowniaceae and Orobanchaceae trib. Lindenbergieae.

Data on pollen morphology also confirm the updated phylogeny-based taxonomic circumscription of Cymbarieae as outlined by Fischer (2004) and Olmstead (2016).

\section{Acknowledgements}

The authors express their gratitude to James C. Solomon, Head Curator of the Missouri Botanical Garden Herbarium (MO; St. Louis, Missouri, USA), Tatyana V. Shulkina, and other staff members of MO, and to Nataliya M. Shyian, Head Curator of the National Herbarium of Ukraine (KW; herbarium of the M.G. Kholodny Institute of Botany, National Academy of Sciences of Ukraine), for their cooperation and assistance in extracting pollen samples from herbarium specimens. Kind help and cooperation of Dmytro O. Klymchuk, Head of the Center of Electron Microscopy, and Vitaliy I. Sapsay, SEM operator (M.G. Kholodny Institute of Botany, National Academy of Sciences of Ukraine), is greatly appreciated. The authors are grateful to anonymous reviewers for their detailed review of the manuscript, valuable comments and suggestions.

\section{REFERENCES}

Bennett J.R., Mathews S. Phylogeny of the parasitic plant family Orobanchaceae inferred from phytochrome A. Amer. J. Bot., 2006, 93: 1039-1051.

Chen Zhiyan. Preliminary study of the pollen morphology of Paulownia. J. Wuhan Bot. Res., 1983, 1: 144-146.

Cusimano N., Wicke S. Massive intracellular gene transfer during plastid genome reduction in nongreen Orobanchaceae. New Phytol. 2016, 210: 680-693. https://doi.org/10.1111/nph.13784.

Doweld A. Prosyllabus Tracheophytorum: Tentamen systematis plantarum vascularium (Tracheophyta). Moscow: GEOS, 2001, 1xxx + 110 pp. [In Russian and English: Доуэльд А.Б. Prosyllabus Tracheophytorum: onыm системы сосудистых растений (Tracheophyta). М.: ГЕОC, 2001, lxxx + $110 \mathrm{c}$.$] .$

Erdtman G. Pollen morphology and plant taxonomy. Angiosperms. Stockholm: Almqvist \& Wiksell, 1952, 539 pp.

Fischer E. Scrophulariaceae. In: The families and genera of vascular plants. Ed. K. Kubitzki. Berlin; Heidelberg; New York: Springer, 2004, pp. 333-432.

Hjertson M.L. Taxonomy, phylogeny and biogeography of Lindenbergia (Scrophulariaceae). Bot. J. Linnean Soc., 1995, 179: 265-321. https://doi. org/10.1016/S0024-4074(95)80002-6.

Inceoğlu Ö. Pollen grains in some Turkish Rhinantheae (Scrophulariaceae). Grana, 1982, 21: 83-96. https://doi. org/ 10.1080/00173138209427684.

Lu L., Wang H., Blackmore S., Li D.-Z., Dong L.-N. Pollen morphology of the tribe Rhinantheae (Orobanchaceae) and its systematic significances. Pl. Syst. Evol., 2007, 268: 177-198. https://doi.org/10.1007/s00606-007-0562-x.

Mabberley D.J. The plant-book: a portable dictionary of the vascular plants. Ed. 2, Cambridge: Cambridge Univ. Press, 1997, 858 pp.

Minkin J.P., Eshbaugh W.H. Pollen morphology of the Orobanchaceae and rhinanthoid Scrophulariaceae. Grana, 1989, 28: 1-18. https://doi. org/10.1080/00173138909431007. 
McNeal J.R., Bennett J.R., Wolfe A.D., Mathews S. Phylogeny and origins of holoparasitism in Orobanchaceae. Amer. J. Bot., 2013, 100: 971-983. https://doi.org/ 10.3732/ajb. 1200448.

Mosyakin S.L., Tsymbalyuk Z.M. Pollen morphology of the southern African tribe Teedieae, an early-branching lineage of crown Scrophulariaceae. Willdenowia, 2015a, 45: 65-75. https://doi.org/10.3372/wi.45.45107.

Mosyakin S.L., Tsymbalyuk Z.M. Pollen morphology of the tribes Aptosimeae and Myoporeae supports the phylogenetic pattern in early-branching Scrophulariaceae revealed by molecular studies. Willdenowia, 2015b, 45: 209-222. https://doi.org/10.3372/wi.45.45207.

Mosyakin S.L., Tsymbalyuk Z.M. Pollen morphology of the tribe Hemimerideae: possible evidence of ancestral pollen types and parallel evolution in the basalmost clade of Scrophulariaceae s. str. Willdenowia, 2017, 47: 15-27. https://doi.org/10.3372/wi.47.47102.

Olmstead R.G., DePamphilis C.W., Wolfe A.D., Young N.D., Elisons W.J., Reeves P.A. Disintegration of the Scrophulariaceae. Amer. J. Bot., 2001, 88: 348-361.

Olmstead R. (with the help of: D. Albach, P. Beardsley, D. Bedigian et al.). A synoptical classification of the Lamiales. Version 2.6.2 (updated 12 April, 2016), available at: http://depts.washington.edu/phylo/Classification.pdf.

Piwowarczyk R., Madeja J., Nobis M. Pollen morphology of the Central European broomrapes (Orobanchaceae: Orobanche, Phelipanche and Orobanchella) and its taxonomical implications. Pl. Syst. Evol., 2015, 301(2): 795-808. https://doi.org/10.1007/s00606-014-1117-6.

Prijanto B. The Asiatic species of Lindenbergia Lehm. (Scrophulariaceae). Reinwardtia, 1969, 7: 543-560.

Punt W., Hoen P.P., Blackmore S., Nilsson S., Le Thomas A. Glossary of pollen and spore terminology. Rev. Palaeobot. Palynol., 2007, 143: 1-81. https://doi. org/10.1016/j.revpalbo.2006.06.008.

Reveal J.L. An outline of a classification scheme for extant flowering plants. Phytoneuron, 2012, 2012-37: 1-221.

Schäferhoff B., Fleischmann A., Fischer E., Albach D.C. Borsch T., Heubl G., Müller K.F. Towards resolving Lamiales relationships: insights from rapidly evolving chloroplast sequences. BMC Evol. Biol., 2010, 10: 352374. https://doi.org/10.1186\%2F1471-2148-10-352.

Schneider A.C. Resurrection of the genus Aphyllon for New World broomrapes (Orobanche s. 1., Orobanchaceae). PhytoKeys, 2016, 75: 107-118. https://doi. org/10.3897/phytokeys.75.10473.

Schneider A.C., Colwell A.E.L., Schneeweiss G.M., Baldwin B.G. Cryptic host-specific diversity among western hemisphere broomrapes (Orobanche s. 1., Orobanchaceae). Ann. Bot., 2016, 118: 1101-1111. https://doi.org/10.1093/aob/mcw158.

Schneider A.C., Moore A.J. Parallel amphitropical disjunctions of a parasitic plant and its host. Amer. J. Bot., 2017, 104: 1745-1755. https://doi.org/10.3732/ajb.1700181.

Stevens P.F. Angiosperm Phylogeny Website. 2001-onwards, Version 14, July 2017 [and more or less continuously updated since], available at: http://www.mobot.org/MOBOT/research/APweb/, accessed 12.03.2018.

Tank D.C., Beardsley P.M., Kelchner S.A., Olmstead R.G. Review of the systematics of Scrophulariaceae s. 1. and their current disposition. Austral. Syst. Bot., 2006, 19: 289-307. https://doi.org/10.1071/SB05009.

Takhtajan A.L. Systema Magnoliophytorum. Leningrad: Nauka, 1987, 439 рр. [Тахтаджян А.Л. Система магнолиофитов. Л.: Наука, 1987, 439 с.].

Takhtajan A.L. Diversity and classification of flowering plants. New York: Columbia Univ. Press, 1997, 663 pp.

Takhtajan A. Flowering Plants. Berlin: Springer Verlag, 2009, xlv + 871 pp. https://doi.org/10.1007/978-1-4020-9609-9.

Tokarev P.I. Morphology and ultrastructure of the pollen grains. Moscow: KMK Scientific Press, 2002, 51 pp. [Токарев П.И. Морфология и ультраструктура пыльцевых зерен. М.: Т-во науч. изд. КМК, 2002, 51 с.].

Tsymbalyuk Z.M. Ukr. Bot. J., 2011, 68(1): 45-57. [Цимбалюк 3.М. Порівняльне паліноморфологічне дослідження деяких родів Orobanchaceae флори України. Укр. бот. журн., 2011, 68(1): 45-57].

Tsymbalyuk Z.M. Ukr. Bot. J., 2014, 71(6): 660-664. [Цимбалюк 3.M. Філогенетичне положення роду Paulownia: порівняльний аналіз паліноморфологічних свідчень. Укр. бот. журнн., 2014, 71(6): 660-664]. https://doi.org/10.15407/ukrbotj71.06.660.

Tsymbalyuk Z.M. Palynomorphological peculiarities of representatives of the order Lamiales s. l.: phylogenetic significance and main trends of evolution: Dr. Sci. Diss. Kуiv, 2016, 449 рр. [Цимбалюк 3.М. Паліноморфологічні особливості представників порядку Lamiales s.l.: філогенетичне значення та напрямки еволюції: дис. ... д-ра біол. наук: спец. 03.00.05 "Ботаніка", Київ, 2016, 449 c.]. https://doi.org/10.13140/rg.2.2.17133.79843.

Tsymbalyuk Z.M., Mosyakin S.L. Atlas of pollen grains of representatives of Plantaginaceae and Scrophulariaceae. Kyiv: Nash Format, 2013a, 276 pp. [Цимбалюк 3.M., Мосякін С.Л. Атлас пилкових зерен представників родин Plantaginaceae та Scrophulariaceae. Київ: Наш формат, 2013a, 276 c.]. https://doi.org/10.13140/ RG.2.2.16968.11527.

Tsymbalyuk Z.M., Mosyakin S.L. Ukr. Bot. J., 2013b, 70(5): 600-609. [Цимбалюк 3.M., Мосякін С.Л. Паліноморфологія видів Orobanche L. subgen. Phelipanche (Pomel) Tzvelev (Orobanchaceae) флори України. Укр. бот. жмурн., 2013b, 70(5): 600-609]. https://doi.org/10.15407/ukrbotj70.05.600

Tsymbalyuk Z.M., Mosyakin S.L. Ukr. Bot. J., 2013c, 70(6): 723-731. [Цимбалюк 3.М., Мосякін С.Л. Паліноморфологія видів Orobanche L. підроду Orobanche (Orobanchaceae) флори України. Укр. бот. жмурн., 2013c, 70(6): 723-731]. https://doi.org/10.15407/ ukrbotj71.04.442.

Tsymbalyuk Z.M., Mosyakin S.L. Ukr. Bot. J., 2014, 71(4): 442-448. [Цимбалюк 3.M., Мосякін С.Л. Еволюційно-паліноморфологічний аналіз деяких триб родини Plantaginaceае. Укр. бот. журн., 2014, 71(4): 442-448]. https://doi.org/10.15407/ukrbotj71.04.442. 
Tsymbalyuk Z.M., Mosyakin S.L. Ukr. Bot. J., 2017, 74(4): 310-325. [Цимбалюк 3.М., Мосякін С.Л. Паліноморфологічні особливості представників триби Rhinantheae (Orobanchaceae) у світлі молекулярнофілогенетичних даних. Укр. бот. жмурн., 2017, 74(4): 310-325]. https://doi.org/10.15407/ukrbotj74.04.310.

Wei Zh.X. Pollen morphology of Wightia and its taxonomic significance. Acta Bot. Yunnan., 1989, 11(1): 65-70. [In Chinese, with English abstract].

Zare G., Dönmez A.A., Dönmez E.O. Pollen morphology and evolution in the genus Orobanche L. s. 1. and its allied genera (Orobancheae/Orobanchaceae) in Turkey. Pl. Syst. Evol., 2014, 300(5): 783-802. https://doi. org/10.1007/s00606-013-0919-2.

Zhou Q.-M., Jensen S.R., Liu G.-L., Wang Sh., Li H.-Q. Familial placement of Wightia (Lamiales). Pl. Syst. Evol., 2014, 300(9): 2009-2017. https://doi.org/10.1007/ s00606-014-1029-5.

Recommended for publication by D.V. Dubyna

Submitted 10.02.2018

Цимбалюк 3.М., Мосякін С.Л. Паліноморфологічні особливості представників триб Lindenbergieae i Cymbarieaе та еволюція пилку в базальних кладах Orobanchaceae.

Укр. бот. журн., 2018, 75(2): 123-136.

Інститут ботаніки ім. М.Г. Холодного НАН України вул. Терещенківська, 2, Київ 01004, Україна

3 використанням світлової та сканувальної електронної мікроскопії досліджено морфологію пилкових зерен чотирьох видів 3 чотирьох родів триби Cymbarieae та двох видів роду Lindenbergia триби Lindenbergieae (Orobanchaceae). Встановлено, що пилкові зерна триби Lindenbergieae 3-борозно-орові (зрідка 2-борозно-орові), еліпсоїдальні, сфероїдальні або сплющено-сфероїдальні за формою, дрібних розмірів, з сітчастою скульптурою екзини. Пилкові зерна триби Cymbarieae 3-борозні, зрідка 4-борозні, еліпсоїдальні, сфероїдальні або сплющеносфероїдальні за формою, середніх або великих розмірів, 3 сітчасто-паличковою або зморшкувато-сітчасто-паличковою скульптурою екзини. Особливості пилкових зерен представників триб Cymbarieae i Lindenbergieae порівнюються 3 такими в Paulowniaceae і верхівкових кладах Orobanchaceae. Базальна клада Orobanchaceae (Lindenbergieae) за паліноморфологічними особливостями подібна до Paulowniaceae (сестринська клада до всіх Orobanchaceae) і Wightia. Паліноморфологічні висновки підтверджують філогенетичні закономірності, які виявлені у базальних Orobanchaceae та їхніх найближчих родичів. Зроблено висновок, що борозно-оровий (ймо- вірно, 3-борозно-оровий) тип пилкових зерен може бути предковим у родині Orobanchaceae. Різноманітність пилкових зерен у верхівкових кладах Orobanchaceae еволюціонувала на основі декількох типів і підтипів пилку, властивих гіпотетичним предкам Orobanchaceae; такі типи, ймовірно, збереглися в сучасних представників Paulowniaceae та Lindenbergieae.

Ключові слова: Cymbarieae, Lindenbergieae, Orobanchaceae, еволюція пилку, морфологія пилку, систематика, філогенія

Цымбалюк 3.Н., Мосякин С.Л. Палиноморфологические особенности представителей триб Lindenbergieae и Cymbarieaе и эволюция пылыцы в базальных кладах Orobanchaceae. Укр. бот. журн., 2018, 75(2): 123-136.

Институт ботаники им. Н.Г. Холодного НАН Украины ул. Терещенковская, 2, Киев 01004, Украина

С помощью световой и сканирующей электронной микроскопии изучены пыльцевые зерна четырех видов из четырех родов трибы Cymbarieae и двух видов рода Lindenbergia трибы Lindenbergieae (Orobanchaceae). Установлено, что пыльцевые зерна трибы Lindenbergieae 3-бороздно-оровые (изредка 2-бороздно-оровые), эллипсоидальные, сфероидальные или сплющено-сфероидальные по форме, мелких размеров, с сетчатой скульптурой экзины. Пыльцевые зерна трибы Cymbarieae 3-бороздные, изредка 4-бороздные, эллипсоидальные, сфероидальные или сплющено-сфероидальные по форме, средних или больших размеров, с сетчато-палочковой или морщинисто-сетчасто-палочковой скульптурой экзины. Особенности пыльцевых зерен представителей триб Cymbarieae и Lindenbergieae сравниваются с таковыми у Paulowniaceae и терминальных клад Orobanchaceae. Базальная клада в семействе Orobanchaceae (Lindenbergieae) по палиноморфологическим особенностям сходна с Paulowniaceae (сестринская клада по отношению к Orobanchaceae) и Wightia. Палиноморфологические данные подтверждают филогенетические закономерности, выявленные в настоящее время у базальных Orobanchaceae и их ближайших родственников. Сделан вывод о том, что бороздно-оровый (возможно, 3-бороздно-оровый) тип пыльцевых зерен может быть предковым в семействе Orobanchaceae. Разнообразие пыльцы Orobanchaceae развилось на основе нескольких типов и подтипов, которые были свойственны гипотетическим предкам Orobanchaceae и, вероятно, сохранились у современных представителей Paulowniaceae и Lindenbergieae.

Ключевые слова: Cymbarieae, Lindenbergieae, Orobanchaceae, эволюция пыльцы, морфология пыльцы, систематика, филогения 